\title{
Manual discrimination of compliance using active pinch grasp: The roles of force and work cues
}

\author{
HONG Z. TAN, NATHANIEL I. DURLACH, \\ G. LEE BEAUREGARD, and MANDAYAM A. SRINIVASAN \\ Massachusetts Institute of Technology, Cambridge, Massachusetts
}

\begin{abstract}
In these experiments, two plates were grasped between the thumb and the index finger and squeezed together along a linear track. The force resisting the squeeze, produced by an electromechanical system under computer control, was programmed to be either constant (in the case of the force discrimination experiments) or linearly increasing (in the case of the compliance discrimination experiments) over the squeezing displacement. After completing a set of basic psychophysical experiments on compliance resolution (Experiment 1), we performed further experiments to investigate whether work and/or terminal-force cues played a role in compliance discrimination. In Experiment 2, compliance and force discrimination experiments were conducted with a roving-displacement paradigm to dissociate work cues (and terminal-force cues for the compliance experiments) from compliance and force cues, respectively. The effect of trial-by-trial feedback on response strategy was also investigated. In Experiment 3, compliance discrimination experiments were conducted with work cues totally eliminated and terminal-force cues greatly reduced. Our results suggest that people tend to use mechanical work and force cues for compliance discrimination. When work and terminal-force cues were dissociated from compliance cues, compliance resolution was poor (22\%) relative to force and length resolution. When work cues were totally eliminated, performance could be predicted from terminal-force cues. A parsimonious description of all data from the compliance experiments is that subjects discriminated compliance on the basis of terminal force.
\end{abstract}

To a first approximation, the mechanical behavior of all deformable solid objects can be expressed as $f=F_{s}+$ $K x+B \dot{x}+m \ddot{x}$, which represents the relationship between the total force $(f)$ applied on the object and the corresponding displacement $(x)$, velocity $(\dot{x})$, and acceleration $(\ddot{x})$; the frictional force $\left(F_{s}\right)$, linear stiffness $(K)$, viscosity $(B)$, and mass $(M)$ are the physical parameters that distinguish one object from another (we use lowercase letters for variables and uppercase for parameters). It is our goal to study manual resolution of all these physical variables and parameters and to provide basic psychophysical information that can be used to (1) advance our understanding of manual perception of object properties, (2) guide the development of design specifications for haptic interfaces that not only sense position and force commands from the human operator but also display such information to the operator in teleoperation and virtual environment systems (see, e.g., the recently published book on systems of this type edited by Durlach \& Mavor, 1994), and (3) improve the design of autonomous

This work was supported in part by Grant 5-R01-DC00126 from the National Institutes of Health, in part by Grant N00014-90-J-1935 from the Office of Naval Research, and in part by Contract No. N61339-93C-0083 from the Naval Air Warfare Center Training Systems Division. The authors wish to thank William Rabinowitz and Lynette Jones for their extensive help with the revision of the original manuscript. Correspondence should be addressed to H. Z. Tan, Massachusetts Institute of Technology, Room 36-755, Sensory Communication Group, Research Laboratory of Electronics, Cambridge, MA 02139-4307 (e-mail: tan@ cbgrle.mit.edu). robots that must make use of manual sensing and manipulation.

This is the third in a series of papers concerned with how individual physical properties of objects are perceived. In the first paper of this series (Durlach et al., 1989), we reported the results of a variety of experiments in which the subject was required to discriminate or identify object length (related to the variable $x$ ) by means of the fingerspan method. The just noticeable difference (JND) in length measured in discrimination experiments was roughly $1 \mathrm{~mm}$ for reference lengths of $10-20 \mathrm{~mm}$, and increased to roughly $2.2 \mathrm{~mm}$ for a reference length of $80 \mathrm{~mm}$. The results violated Weber's law. In the second paper (Pang, Tan, \& Durlach, 1991), we discussed experiments in which the subject was required to discriminate resistive force (represented by the parameter $F_{s}$ ) by means of active finger motion. The JND was found to be $7 \%$ of the reference force over a reference force range of $2.5-10.0 \mathrm{~N}, 1$ a displacement range of 5-30 mm, an initial finger-span range of 45$125 \mathrm{~mm}$, and a mean velocity range of $25-160 \mathrm{~mm} / \mathrm{sec}$. These results were roughly consistent with those reported for weight and force by other researchers (e.g., Jones, 1989; see also the review by Jones, 1986).

Manual perception of stiffness $K$ (i.e., change in force divided by change in displacement) or, equivalently, compliance $1 / K$ (i.e., change in displacement divided by change in force) is presumably based on the perception of both force and displacement, since humans possess no known special "stiffness/compliance sensors" in the peripheral sensory organs. During active touch, although both tac- 
tile $^{2}$ and kinesthetic (also called proprioceptive) sensory systems of the hand can convey force information, only the kinesthetic system is able to convey information about the overall displacement of the finger. In order to infer the actual displacement imposed on the object, further correction to the finger displacement may need to be applied to account for the fingerpad deformation. Much of the research on the kinesthetic system has focused on the relative contributions of the mechanoreceptors in the skin, joint, and muscle to the perception of position, movement, and force (e.g., see the review by Clark \& Horch, 1986). A comprehensive and accurate characterization of stiffness or compliance resolution could provide useful data for the evaluation of hypotheses concerning the mechanisms that underlie the sense of stiffness or compliance.

In a series of experiments involving perception of objects attached to the wrist through activation of the elbow joint, Jones and Hunter (Jones, 1989; Jones \& Hunter, $1990,1992 \mathrm{a}, 1992 \mathrm{~b}, 1993$ ) have systematically examined human resolution of movement, force, stiffness, and viscosity. They have found that the JND for viscosity $(34 \%)$ is 1.5 times that for stiffness ( $23 \%$ ), which, in turn, is about 3 times those for force, position, and movement $(7 \%-8 \%)$. The results were interpreted as evidence that there is a loss in perceptual resolution when the discrimination task requires force and displacement or velocity cues to be combined. In recent experiments involving active and passive touch with and without local anesthesia to the fingerpads, Srinivasan and LaMotte (1994) have shown that the mechanisms of compliance discrimination depend on whether the compliant objects have rigid or deformable surfaces. The discrimination of the softness of rubber specimens (i.e., compliant objects with deformable surfaces) is accomplished through purely tactile information mediated by the mechanoreceptors in the fingerpad skin, whereas the discrimination of the compliance of rigid surfaces supported by springs requires kinesthetic information. This latter result supports the conclusion of Roland and Ladegaard-Pedersen (1977), who examined the stiffness NND by asking subjects to compress springs held between the thumb and the index finger. They found, using skin and joint anaesthesia, that the ability to discriminate spring stiffness was not affected by the lack of these sensory signals from the fingers compressing the springs (i.e., even reduced kinesthetic information is sufficient for stiffness discrimination).

At a more basic level, it has been documented that the perception of the physical variables position, movement, and force are not independent in the kinesthetic system. For example, Clark, Burgess, and Chapin (1986) demonstrated that whereas a small change in the metacarpophalangeal joint angle can be readily detected even at an extremely slow velocity (i.e., $0.016^{\circ} / \mathrm{sec}$ ), the perception of a change in the position of the proximal interphalangeal (PIP) joint is very much dependent upon the velocity. Ferrell and Milne (1989) found, using a finger matching paradigm in which the subject actively positioned one (matching) finger until its PIP joint position was per- ceived to be the same as that of the other (target) finger, that matching errors in PIP joint position increase with decreasing velocity of the displacement of the target finger during digital nerve block of that finger, but not with an unanesthetized target finger. Thus, at least in some joints, the sense of position is influenced by movement cues under certain conditions. In another study, Watson, Colebatch, and McCloskey (1984) reported that the error in matching the position of the left index finger with the right one increased monotonically with the stiffness of the elastic load applied at the right wrist. However, when asked to match force instead of position, the errors were essentially independent of the relative position of the two fingers. Their results indicate that the perceptual interaction between force and position is not symmetrical: force cues have a bigger influence in altering the perception of position than position cues do on force perception. Given these results, it is reasonable to speculate that the perception of compliance or stiffness may also be influenced by force and/or displacement cues, or a combination of both (e.g., mechanical work, which is defined as force integrated over displacement). In this study, we examined the dependence of compliance resolution on the perceptual cues that are available to be utilized in such a task. Specifically, we studied the relative roles played by force and mechanical-work cues during manual compliance discrimination using active pinch grasp.

The remainder of this paper includes a General Method section in which the common elements for all experiments are presented, followed by detailed discussions of each experiment. The paper then concludes with a general discussion of our findings and further comments on the relation of these findings to other work. Partial results from this series of experiments have been summarized in Tan, Pang, and Durlach (1992) and Tan, Durlach, Shao, and Wei (1993).

\section{GENERAL METHOD}

\section{Apparatus}

The apparatus used in the compliance experiments, shown in Figure 1, was the same as that used for force discrimination experiments reported in Pang et al. (1991). The device had two parallel aluminum plates: one was fixed, and the other could be moved along a linear track perpendicular to the plates. A cylindrical roller was mounted on the movable plate to serve as the contact point for the thumb so that (1) active and reactive forces were always applied perpendicular to the plate, and (2) readings from a strain gauge mounted at the bottom of the movable plate could be accurately converted to force values. In this paper, the direction of active motion is called the positive direction and the opposite is called the negative direction (see Figure 2). At the beginning of a trial, the movable plate was placed against the left mechanical stop and kept at $x=0$. The subject then grasped the two plates between the fingertips of the thumb and index finger and squeezed the movable plate horizontally toward the fixed plate. During the squeeze, the motor driving the movable plate applied a resistive force to the thumb in the negative direction. The control algorithm was capable of delivering any predefined force versus displacement profile during that period. When the movable plate impacted the mechanical wall (marked $x=X_{\text {wall }}$ in Figure 2), the force applied to the movable plate 


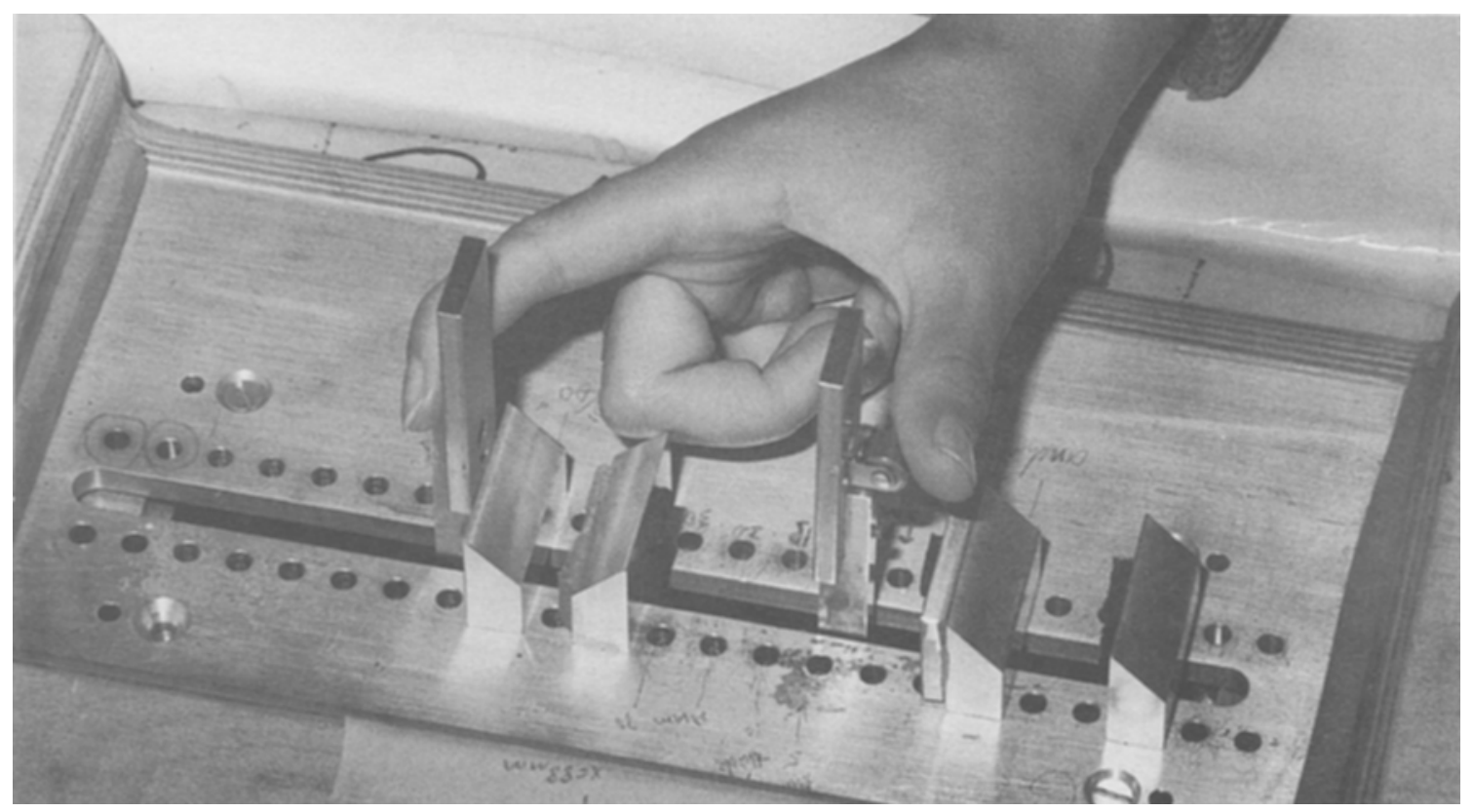

Figure 1. Electromechanical device used to study compliance perception.

by the motor was instantaneously changed to the positive direction so that the movable plate was pushed against the mechanical wall, and the thumb could be removed from the roller.

After the subject entered the response, the movable plate was brought back to the resting position at $x=0$. This completed one trial. Thin foam pieces were placed on the mechanical stops to absorb any vibration caused by the impact of the movable plate. Note that the positions of the three mechanical stops were not fixed. They could be inserted into any one of the holes that were placed $5 \mathrm{~mm}$ apart along the linear track. For the compliance experiments, the device simulated a compliant object with rigid surfaces (i.e., the two parallel plates) that can be modeled by a linear spring [i.e., $\left.f(x)=F_{s}+x / C\right]$. We describe the object as an unloaded spring when $F_{s}=0$, and as a preloaded spring when $F_{s}>0$. For the force experiments, the device simulated a constant force [i.e., $f(x)=F_{s}$ was independent of the displacement $x$ ].

\section{Subjects}

Eight subjects, 3 males (S2, S6, and S8) and 5 females, 19--54 years old, participated in our study. S1-S3 also served in the force discrimination experiments reported in Pang et al. (1991). S1 and S4-S7 were research staff, S2 was blind and hearing-impaired, and S3 and S8 were graduate and undergraduate students, respectively, at MIT. S2, S3, and S8 were paid on an hourly basis. All subjects were right-handed with no known hand impairments and used the right hand for all experiments.

\section{Procedure}

The standard one-interval two-alternative forced-choice paradigm was employed for all experiments. Trial-by-trial correct-answer feedback was given during all experiments except Experiment 2C. (Initial training was provided to subjects in Experiment $2 \mathrm{C}$ to make sure that they understood the instructions.) There were two admissible stimuli: reference, $C_{0}$, and reference plus increment, $C_{0}+\Delta C$, for compliance discrimination $\left(F_{0}\right.$ and $F_{0}+\Delta F$ for force discrimination in Experiment $2 \mathrm{~B}$ ). There were two admissible responses: "1" and "2." On each trial, one of the two stimuli was presented randomly with an a priori probability of .5 . The subject was in- structed to respond " 1 " for $C_{0}+\Delta C$, the "softer spring," and " 2 " for $C_{0}$, the "stiffer spring" (for force discrimination, " 1 " for $F_{0}+\Delta F$, the "larger force," and " 2 " for $F_{0}$, the "smaller force"). Each experimental run consisted of 64 trials. The initial finger span between the thumb and the index finger (see Figure 2) was always kept at $105 \mathrm{~mm}$. The subject's elbow and wrist were always supported, and earplugs were worn to eliminate any possible auditory cues. The computer monitor was placed on the left side of the subject; thus, the subject looked away from the apparatus during the experiments. Experimental sessions typically lasted $1-2 \mathrm{~h}$, and the subject could take a break between runs whenever needed. The subjects were tested 3-5 times a week over the time period during which the experiments were conducted.

In some experiments (Experiments 1 and 3), the fixed-displacement paradigm was used (i.e., the displacement of each squeeze was kept constant within an experimental run). The position of the mechanical wall (see Figure 2) was changed for each experimental run to achieve the desired displacement $X$. Terminal force referred to the resistive force at displacement $X$ right before the movable plate impacted the mechanical wall. In the other experiments (Experiments $2 \mathrm{~A}, 2 \mathrm{~B}$, and $2 \mathrm{C}$ ), the roving-displacement paradigm was used (i.e., the displacement was randomized from trial to trial- -or, equivalently, squeeze to squeeze). The resistive force was dropped to zero at $x=X\left(0<X<X_{\text {wall }}\right)$ to achieve an effective displacement of $X$ (see Figure 2). In this case, terminal force referred to the resistive force at displacement $X$ before it went down to 0 . As reported in Pang et al. (1991), the force JND is essentially the same for the two methods of termination. Our preliminary testing showed that the same was also true for compliance discrimination.

\section{Data Analysis}

Data from each experimental run of 64 trials formed a $2 \times 2$ stimulus-response matrix. The sensitivity index $d^{\prime}, \mathrm{NND}$, and response bias $\beta$ were derived from these matrices to characterize the results (e.g., see Berliner \& Durlach, 1973). In this method of data processing, it is assumed that the underlying density functions associated with the two stimuli being discriminated are normal and of equal variance (means $M_{1}$ and $M_{2}$, and variance $\sigma^{2}$ ). The sensi- 


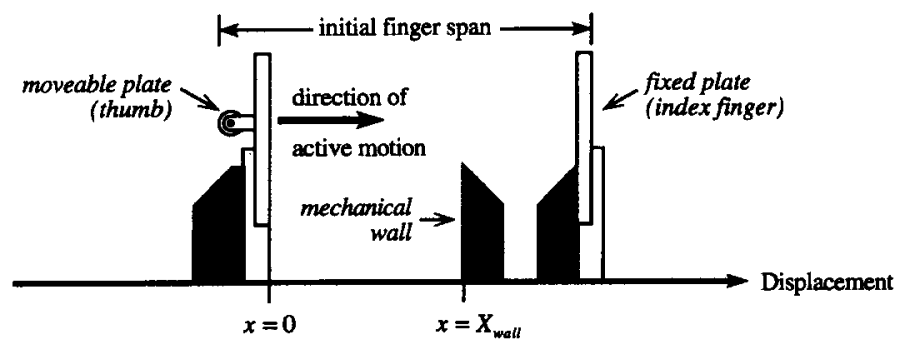

Figure 2. Diagram illustrating the layout of the apparatus.

tivity index $d^{\prime}$ is then defined as the normalized difference between the means; that is,

$$
d^{\prime}=\left(M_{2}-M_{1}\right) / \sigma
$$

The bias $\beta$ is defined as the normalized deviation of the response criterion $(Q)$ from the midpoint between the two means; that is,

$$
\beta=\left[Q-\left(M_{1}+M_{2}\right) / 2\right] / \sigma .
$$

Therefore, the condition $\beta=0$ corresponds to unbiased response behavior.

Generally speaking, the values of $d^{\prime}$ were found (as in auditory studies) to be roughly proportional to the increment $\Delta C / C_{0}$ (or $\left.\Delta F / F_{0}\right)$. Given this proportionality, performance can be summarized by the slope $\delta=d^{\prime} /\left(\Delta C / C_{0}\right)$ [or $\delta=d^{\prime} /\left(\Delta F / F_{0}\right)$ ] averaged over the different values of $\Delta C / C_{0}$ (or $\left.\Delta F / F_{0}\right)$ tested for the same $C_{0}$ (or $\left.F_{0}\right)$. The JND for compliance (or force), $(\Delta C)_{0}\left[\right.$ or $\left.(\Delta F)_{0}\right]$, was defined by the performance threshold $d^{\prime}=1$. The Weber fraction (denoted $\mathrm{JND} \%$ ) at the reference compliance $C_{0}$ (or reference force $F_{0}$ ) was then computed from the average slope $\delta$; that is,

$$
\operatorname{JND} \%=(\Delta C)_{0} / C_{0}\left[\operatorname{or}(\Delta F)_{0} / F_{0}\right]=(1 / \bar{\delta}) \times 100 \% \text {. }
$$

\section{EXPERIMENT 1}

\section{Compliance Discrimination Using the Unloaded- Spring, Fixed-Displacement Paradigm With Feedback}

The paradigm used in Experiment 1 was the most straightforward one to study compliance resolution. It simulated the scenario where a person picks up a compliant object, squeezes it for a fixed displacement, does the same with another object with a different compliance, and compares the compliance values. Although compliance was the main physical variable manipulated in stimulus presentations, other extraneous cues were present. Therefore, Experiment 1 served to reveal the best possible compliance resolution humans could achieve using active finger motion (with possible extraneous cues).

\section{Method}

Three subjects (S1, S2, and S3) participated in Experiment 1. The reference compliance $C_{0}$ was kept at $4 \mathrm{~mm} / \mathrm{N}$, and the increment $\Delta \mathrm{C} / C_{0}$ was set to one of the four values: $5 \%, 10 \%, 15 \%$, or $20 \%$. The subject was instructed to imagine compressing a linear spring between the thumb and index finger and to judge the compliance of the spring. Trial-by-trial correct-answer feedback based on compliance values was provided. The force-displacement profile used for this experiment is shown in Figure 3. The resistive force, $f$, was related to displacement, $x$, by $f=x / C$, where $C$ was either $C_{0}$ or $C_{0}+\Delta C$. The fixed-displacement paradigm was used in this experiment: the total displacement $X$ was set to one of five values for each run: $15,20,25,30$, or $35 \mathrm{~mm}$. There were a total of 20 experimental conditions ( $4 \Delta C / C_{0}$ values $\times 5 X$ values). Each sub- ject was required to perform two runs per condition. The order of the experimental conditions was randomized for each subject.

Note that with this force-displacement profile, the subjects had access to mechanical-work and terminal-force cues as well as compliance cues. In other words, changes in compliance were perfectly correlated with changes in mechanical work and terminal force.

\section{Results}

All the stimulus-response matrices for the same experimental condition (i.e., the same $\Delta C / C_{0}$ and $X$ values) were pooled - that is, the entries in corresponding positions of the matrices obtained from the various runs were summed to form a new matrix representing the overall results for the given condition. From these pooled matrices, the sensitivity index $d^{\prime}$ and the response bias $\beta$ were estimated. The Weber fraction $\mathrm{JND} \%$ was then computed from $d^{\prime}$ values across $\Delta C / C_{0}$ values tested for the same $C_{0}$ and $X$ values.

The response bias $\beta$ was consistently small for all conditions tested in this experiment (i.e., $\beta \leq d^{\prime} / 10$ ). Therefore, Weber fractions completely characterized the data (see the open symbols and the dashed line in Figure 4). According to these data, the compliance JND\% was roughly independent of $X$ over the range $15 \leq X \leq 35 \mathrm{~mm}$, varied between $5 \%$ and $15 \%$ for the subjects tested and averaged $8 \%$ for these subjects.

Note that it was not clear when using this paradigm whether the subject's performance was truly based on compliance cues. In particular, it can be seen (from Figure 3) that $\Delta F / F_{0}=\Delta C /\left(C_{0}+\Delta C\right)$ and, therefore, that when $\Delta C / C_{0}=8 \%, \Delta F / F_{0}=7 \%$. Moreover, according to Pang et al. (1991), the force JND\% is roughly $7 \%$. Thus, the results shown in Figure 4 are consistent with the hypothesis that the subjects selected their responses on the basis of ter-

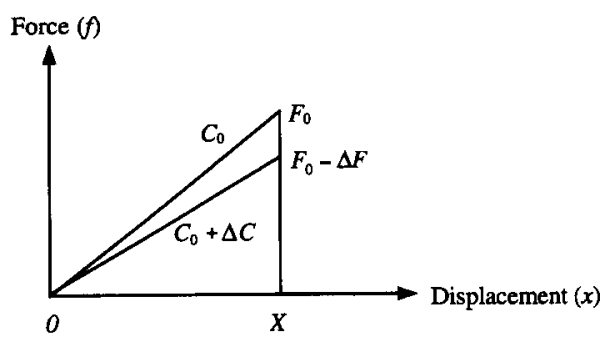

Figure 3. Force-displacement profile for Experiment 1. $C_{0}$ is the reference compliance, $\Delta C$ is the compliance increment, $F_{0}$ is the terminal force associated with $C_{0}, \Delta F$ is the terminal-force increment, and $X$ is the fixed displacement. 


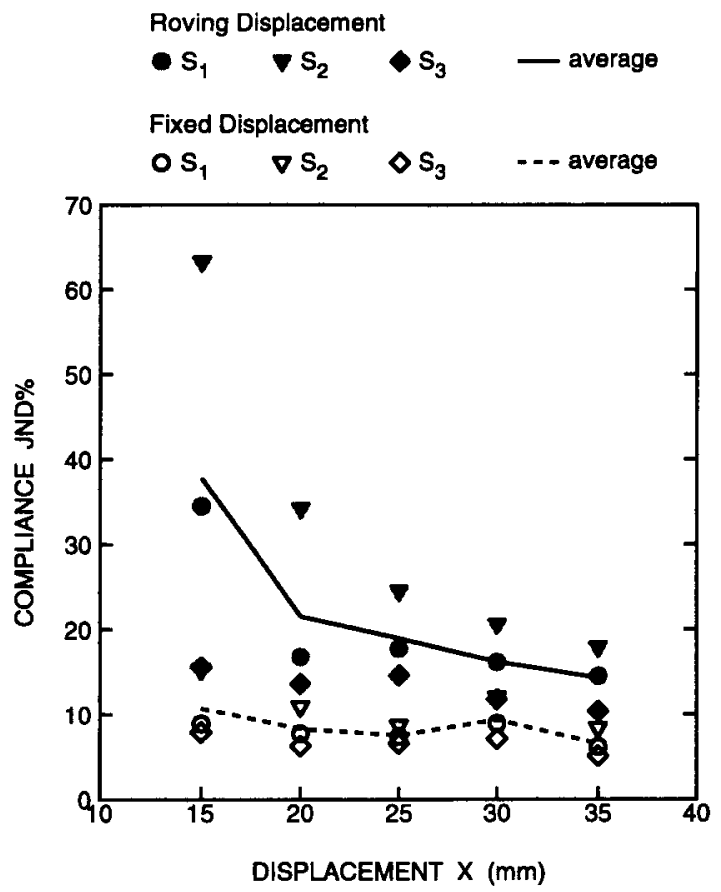

Figure 4. Compliance JND\% results of Experiments 1 (fixed displacement) and $2 \mathrm{~A}$ (roving displacement).

minal force rather than compliance. The subjects could also, of course, have based their responses on the amount of mechanical work [the amount of work $W$ for the two stimuli was $W=F_{0} X / 2$ and $W=\left(F_{0}-\Delta F\right) X / 2$ ]. Unfortunately, work JNDs are not available for comparison. If either terminal force or work was used as cues, then we could not claim to have measured the Weber fractions for compliance.

\section{EXPERIMENT 2}

\section{Compliance and Force Discrimination Using the Roving-Displacement Paradigm}

With the force-displacement profile used in Experiment 1 , a smaller compliance $C_{0}$ was always associated with a larger terminal force $F_{0}$ and more mechanical work
(Figure 3). In Experiment 2, however, the displacement was randomized from trial to trial; thus, it was equally possible for a smaller or larger terminal force to be associated with $C_{0}$. The same was true for mechanical work. If the subjects had focused exclusively on compliance cues in Experiment 1, then their performance with the roving-displacement paradigm in Experiment 2 should not differ greatly from that obtained in Experiment 1. However, if roving-displacement degrades subjects' ability to resolve compliance, then it seems likely that cues other than compliance cues (e.g., work cues or terminal-force cues) were used in Experiment 1.

\section{Experiment 2A: Compliance Discrimination Using the Unloaded-Spring, Roving-Displacement Paradigm With Feedback}

\section{Method}

The same 3 subjects ( $\mathrm{S} 1, \mathrm{~S} 2$, and $\mathrm{S} 3$ ) participated in this experiment. The reference $C_{0}$ was again kept at $4 \mathrm{~mm} / \mathrm{N}$, and the increment $\Delta C / C_{0}$ was set at one of the four values: $10 \%, 20 \%, 30 \%$, or $40 \%$ (the $C_{0}+\Delta C$ values were increased to keep the difficulty of the task at a reasonable level). The subject was again instructed to imagine compressing a linear spring between the thumb and the index finger and to judge the compliance of the spring. Trial-bytrial correct-answer feedback based on compliance values was provided. The force-displacement profiles used in this compliance experiment were essentially the same as in Experiment 1, except that the displacement $X$ was randomized from trial to trial. With this new roving-displacement paradigm (Figure 5), the subjects could no longer rely on the relative magnitudes of terminal force or mechanical work to discriminate compliance correctly. The five displacement values were $X_{1}=15, X_{2}=20, X_{3}=25, X_{4}=30$, and $X_{5}=35 \mathrm{~mm}$, one of which was chosen randomly for each trial with an a priori probability of .2. As far as the subjects were concerned, their task was essentially the same as that in Experiment 1 . With the roving-displacement paradigm, they were still instructed to discriminate $C_{0}$ from $C_{0}+\Delta C$ despite changes in $X$ from trial to trial. The subject's thumb always traveled a distance of $X_{\text {wall }}=40 \mathrm{~mm}$, but the force dropped down to zero for $X_{i}<x \leq X_{\text {wall }}(i=1,2, \ldots, 5)$. Each subject performed 10 runs per $\Delta C / C_{0}$ value. The order of the experimental conditions was randomized for each subject.

\section{Results}

As in Experiment 1, the stimulus-response matrices for the same $\Delta C / C_{0}$ and $X$ values were pooled; from these

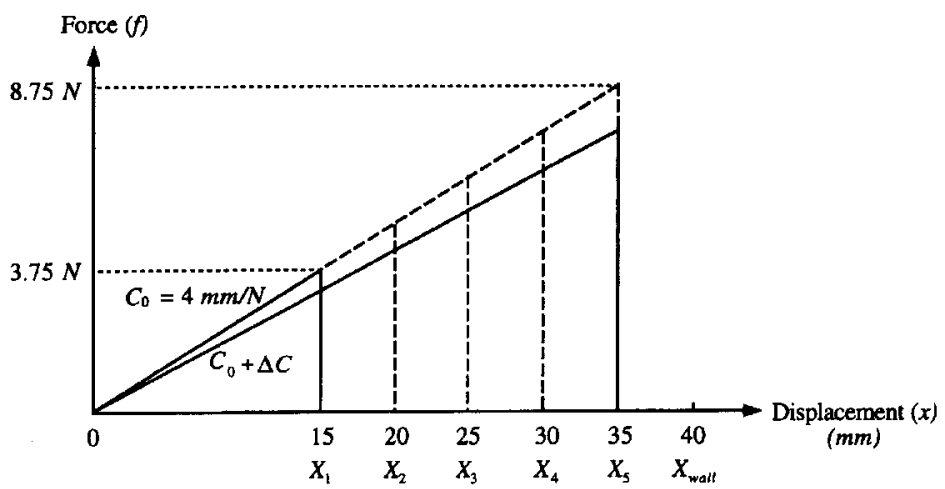

Figure 5. Force-displacement profile for Experiment 2A. Solid lines show two stimuli with $C_{0}$ and $C_{0}+\Delta C$ that differ in displacement (15 and $35 \mathrm{~mm}$, respectively). Dotted lines show the rest of the 10 possible force-displacement profiles associated with $C_{0}, C_{0}+\Delta C$, and $X_{i}(i=1,2, \ldots, 5)$. 
pooled matrices, the sensitivity index $d^{\prime}$ and the response bias $\beta$ were estimated. The Weber fraction JND $\%$ was then computed from $d^{\prime}$ values across $\Delta C / C_{0}$ values tested for the same $C_{0}$ and $X$ values.

The results shown in Figure 4 (the filled symbols and the solid line) indicate that roving $X$ had a strong degrading effect on the ability to discriminate compliance. Whereas the average compliance JND\% from Experiment 1 with fixed-displacement was $8 \%$, the average compliance JND\% from Experiment $2 \mathrm{~A}$ with rovingdisplacement was $22 \%$. Note also that whereas the compliance JND\% from Experiment 1 was essentially independent of $X$, the compliance JND\% from Experiment $2 \mathrm{~A}$ decreased monotonically with $X$.

Unlike the results from Experiment 1, the results from this experiment indicate a significant bias $\beta$ (see the lefthand column in Figure 6). This bias was found to be roughly independent of $\Delta C / C_{0}$ but to depend strongly and in an orderly way on $X$. Specifically, and as shown in the left-hand column of Figure 6 , there was a significant tendency for the bias $\beta$ to be larger when $X$ was small than when $X$ was large. The coding of responses was such that a positive value of $\beta$ corresponds to a tendency to judge the electromechanical spring as soft (i.e., to judge the stimulus to be $C_{0}+\Delta C$ rather than $C_{0}$ ). Note that the data in the left-hand column of Figure 6 indicate a tendency for the subject to judge the spring as soft when $X$ was small and the spring as stiff when $X$ was large. Because the occurrence of relatively small $X$ in this experiment tended to be correlated with relatively small values of mechanical work (and relatively small values of terminal force), these bias results suggest that the subject's responses were influenced by work and/or terminalforce cues. In order to explore this notion more systematically, the analyses described in the next subsections were performed.

The work hypothesis and the $d^{\prime}$ results. The work hypothesis states that the subject responds "stiffer spring" when $W \geq W_{T}$ and "softer spring" when $W<W_{T}$, where $W$, the mechanical work, is given by $W=X^{2} /(2 C)$ and $W_{T}$ is a criterion value of work. Furthermore, it was assumed in evaluating this hypothesis that $W_{T}$ was chosen by the subject to produce roughly the same number of presentations for $W \geq W_{T}$ as for $W<W_{T}$. To the extent that the work hypothesis is correct (i.e., that the subject chose a response on the basis of mechanical work rather than compliance or terminal force), one would expect to obtain a higher $d^{\prime}$ by scoring the responses as correct or incorrect on the basis of work rather than compliance.

In Table 1, column $X$ lists the five displacement values used in the roving-displacement paradigm. Column $\Delta C / C_{0}(\%)$ lists the compliance increments. The refer-

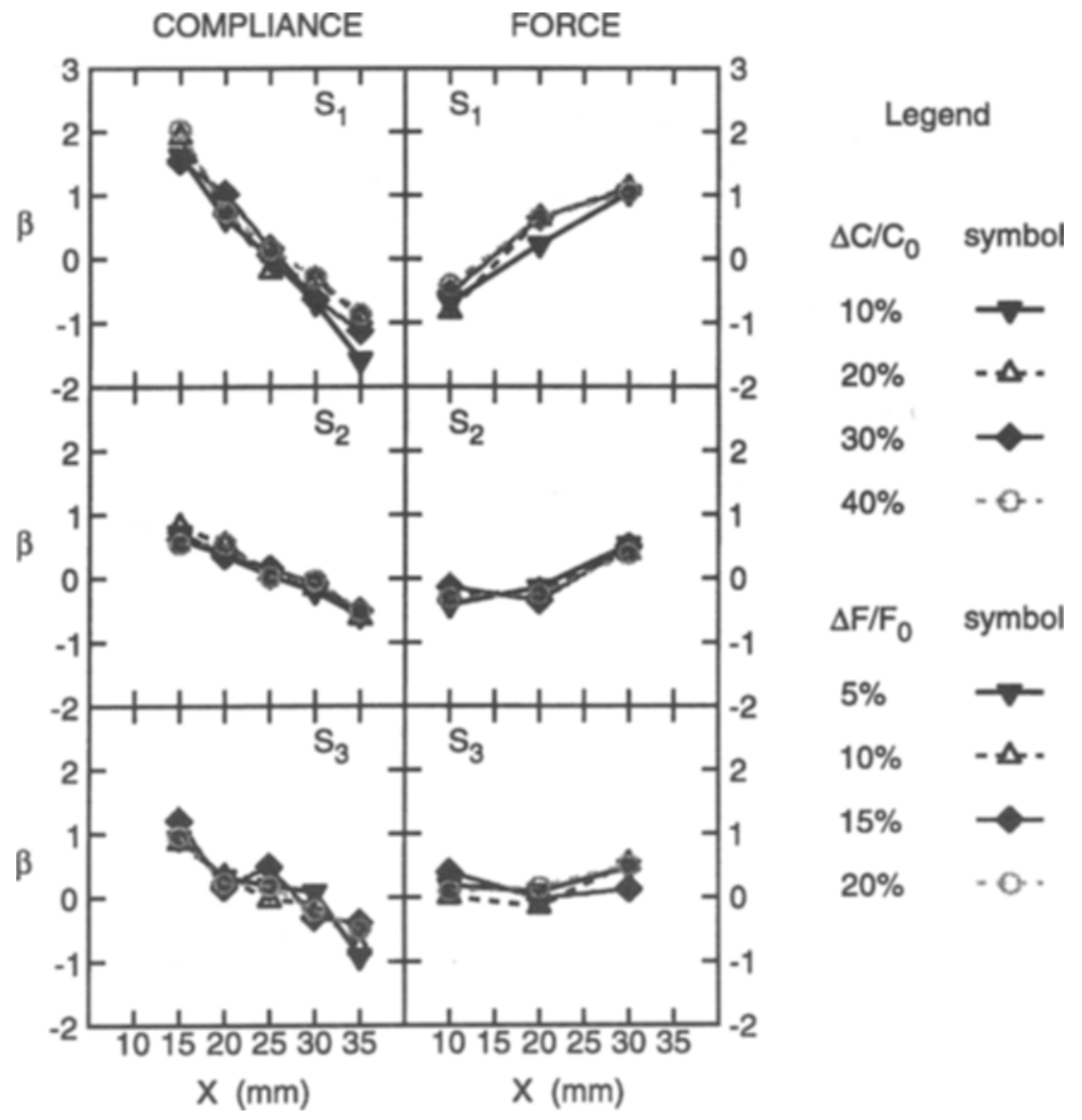

Figure 6. Bias $(\beta)$ results of Experiments $2 A$ and 2B. 
Table 1

The Work Hypothesis and the Relevant Parameters for Experiment $2 \mathrm{~A}$

\begin{tabular}{|c|c|c|c|c|}
\hline $\begin{array}{c}X \\
(\mathrm{~mm})\end{array}$ & $\begin{array}{c}\Delta C / C_{0}(\%) \\
\left(C_{0}=4 \mathrm{~mm} / \mathrm{N}\right)\end{array}$ & $\begin{array}{c}W=X^{2} /\left[2\left(C_{0}+\Delta C\right)\right] \\
(\mathrm{N} \cdot \mathrm{mm})\end{array}$ & $\begin{array}{c}\text { Work } \\
\text { Category }\end{array}$ & $\begin{array}{l}\text { Predicted } \\
\text { Response According } \\
\text { to the Work } \\
\text { Hypothesis }\end{array}$ \\
\hline 15 & $\begin{array}{c}40,30,20, \text { or } 10 \\
0\end{array}$ & $\begin{array}{c}20.1,21.6,23.4,25.6 \\
28.1\end{array}$ & $\begin{array}{l}W<W_{T} \\
W<W_{T}\end{array}$ & $\begin{array}{l}C_{0}+\Delta C \\
C_{0}+\Delta C\end{array}$ \\
\hline 20 & $\begin{array}{c}40,30,20, \text { or } 10 \\
0\end{array}$ & $\begin{array}{c}35.7,38.5,41.7,45.5 \\
50.0\end{array}$ & $\begin{array}{l}W<W_{T} \\
W<W_{T}\end{array}$ & $\begin{array}{l}C_{0}+\Delta C \\
C_{0}+\Delta C\end{array}$ \\
\hline 25 & $\begin{array}{c}40,30,20, \text { or } 10 \\
0\end{array}$ & $\begin{array}{c}55.8,60.1,65.1,71.0 \\
78.1\end{array}$ & $\begin{array}{l}W<W_{T} \\
W>W_{T}\end{array}$ & $\begin{array}{c}C_{0}+\Delta C \\
C_{0}\end{array}$ \\
\hline 30 & $\begin{array}{c}40,30,20, \text { or } 10 \\
0\end{array}$ & $\begin{array}{c}80.4,86.5,93.8,102.3 \\
112.5\end{array}$ & $\begin{array}{l}W>W_{T} \\
W>W_{T}\end{array}$ & $\begin{array}{l}C_{0} \\
C_{0}\end{array}$ \\
\hline 35 & $\begin{array}{c}40,30,20, \text { or } 10 \\
0\end{array}$ & $\begin{array}{c}109.4,117.8,127.6,139.2 \\
153.1\end{array}$ & $\begin{array}{l}W>W_{T} \\
W>W_{T}\end{array}$ & $\begin{array}{l}C_{0} \\
C_{0}\end{array}$ \\
\hline
\end{tabular}

ence compliance, $C_{0}$, is indicated by $\Delta C / C_{0}(\%)=0$ on a separate line for each value of $X$. Column $W$ shows the work associated with the various values of $X$ and $\Delta C / C_{0}(\%)$. Column Work Category puts all the work values shown in column $W$ into two categories: $W<W_{T}$ and $W>W_{T}$, where $W_{T}$ is chosen to produce the same number of presentations for each category. Column Predicted Response shows what the responses would be according to the work hypothesis.

Table 2 shows the values of $d^{\prime}$ derived from the original roving-discrimination data reprocessed according to the work hypothesis. For comparison, the results obtained by defining correct and incorrect responses according to the compliance values (normal processing) and pooling the data over the various values of $X$ are also shown.

For $\mathrm{S} 1$, the $d^{\prime}$ values using the work hypothesis were higher than those based on compliance (except for $\Delta C / C_{0}=$ $40 \%$ ). This supports the hypothesis that work, instead of compliance, was the variable being discriminated by this subject. This hypothesis was also supported by the approximate constancy of $d^{\prime}$ over the variations in $\Delta C / C_{0}$, because the range of $W$ over variations in $X$ was essentially constant for the different $\Delta C / C_{0}$ values (see the $W$ column in Table 1). The $d^{\prime}$ values for $\mathrm{S} 2$ and S3 were much less consistent with the work hypothesis in that some of these values were significantly smaller in the first column than in the second.

The work hypothesis and the $\beta$ results. With the fixeddisplacement paradigm of Experiment 1, there was negligible response bias $\beta$ for compliance discrimination. With the roving-displacement paradigm of Experiment $2 \mathrm{~A}$,

Table 2

Results of Experiment 2A Reprocessed According to the Work Hypothesis

\begin{tabular}{|c|c|c|c|c|c|c|}
\hline \multirow[b]{2}{*}{$\Delta C / C_{0}$} & \multicolumn{3}{|c|}{$\begin{array}{c}d^{\prime} \\
\text { Work Hypothesis }\end{array}$} & \multicolumn{3}{|c|}{$\begin{array}{c}d^{\prime} \\
\text { Normal Processing } \\
(\text { data pooled over } X)\end{array}$} \\
\hline & S1 & S2 & S3 & S1 & S2 & S3 \\
\hline $10 \%$ & 1.63 & 0.79 & 0.77 & 0.26 & 0.28 & 0.63 \\
\hline $20 \%$ & 1.47 & 0.93 & 0.89 & 0.86 & 0.81 & 1.38 \\
\hline $30 \%$ & 1.60 & 0.61 & 0.82 & 1.05 & 1.13 & 2.10 \\
\hline $40 \%$ & 1.41 & 0.91 & 0.90 & 1.65 & 1.25 & 2.39 \\
\hline
\end{tabular}

however, substantial response bias was found (see the lefthand column of Figure 6). These biases were (1) dependent on displacement $X$, and (2) independent of compliance increments $\Delta C / C_{0}$. Both observations are consistent with the work hypothesis. A positive $\beta$ meant that the subject tended to respond "softer spring" too often. According to Figure 6 , when the displacement $X$ was small, the subjects tended to judge the object being squeezed as more compliant. This is consistent with the work hypothesis. The fact that the response bias was independent of $\Delta C / C_{0}$ was also supportive of the work hypothesis, since the difference in the work range resulting from changes in $\Delta C / C_{0}$ was quite small. Among the 3 subjects, the slope of the $\beta$ versus $X$ curve for S1 was twice that for S2, and S3 showed significant biases only at the two extreme values of $X$. Thus, the results for both $\beta$ and $d^{\prime}$ indicate that the performance of S1 was more consistent with the work hypothesis than that of S2 and S3.

The terminal-force hypothesis and the $d^{\prime}$ and $\beta$ results. The terminal-force hypothesis is very similar to the work hypothesis. It states that the subject responds "stiffer spring" when $F \geq F_{T}$ and "softer spring" when $F<F_{T}$, where $F=f(X)$ is the terminal force and $F_{T}$, the criterion value of force, is chosen to produce the same number of presentations for $F \geq F_{T}$ as for $F<F_{T}$. When the results of compliance discrimination experiments are reprocessed using the terminal-force hypothesis, we obtain $d^{\prime}$ values that are exactly the same as those in the left-hand column of Table 2 . The signs of $\beta$ in the left-hand column of Figure 6 are also consistent with the terminal-force hypothesis. In other words, these data and these analyses do not enable us to select either of these hypotheses in preference to the other. This was to be expected, of course, because the terminal-force and mechanical-work cues are totally correlated with each other in this experiment.

\section{Experiment 2B: Force Discrimination Using the Roving-Displacement Paradigm With Feedback}

In view of the strong effect of roving displacement on compliance discrimination, we thought it important to examine the effect of roving displacement on the ability to discriminate force. In the previous experiments on the force JND conducted by Pang et al. (1991), only the 
fixed-displacement paradigm was used. Results obtained from such an experiment might also shed light on whether mechanical-work or terminal-force cues were mainly responsible for the results obtained in Experiment 2A.

\section{Method}

The same 3 subjects ( $\mathrm{S} 1, \mathrm{~S} 2$, and $\mathrm{S} 3$ ) participated in this experiment. The force-displacement profiles used in this experiment are shown in Figure 7. They differ from those in Figure 5 in that resistive force was constant throughout the displacement $X$. The reference $F_{0}$ was kept at $S \mathrm{~N}$, and the increment $\Delta F / F_{0}$ was set to one of the four values: $5 \%, 10 \%, 15 \%$, or $20 \%$. Three displacement values that covered a range similar to those used in Experiment 2A were used: $X_{1}=10, X_{2}=20$, and $X_{3}=$ $30 \mathrm{~mm}$. The value $X_{i}$ was chosen randomly on each trial with an a priori probability of .33 for each of the three cases. The subjects felt a constant resistive force while squeezing the plates and were instructed to discriminate $F_{0}$ from $F_{0}+\Delta F$ despite changes in $X$ from trial to trial. Trial-by-trial correct-answer feedback based on force values was provided. The randomization of $X$ was achieved in a similar way to that in Experiment $2 \mathrm{~A}$. The subject's thumb always traveled a distance of $X_{\text {wall }}=40 \mathrm{~mm}$, but the force dropped down to zero for $X_{i}<x \leq X_{\text {wall }}(i=1,2,3)$. Each subject performed 16 runs per $\Delta F / F_{0}$ value. The order of the experimental conditions was randomized for each subject.

\section{Results}

In a manner analogous to that used in Experiments 1 and $2 \mathrm{~A}$, the stimulus-response matrices for the same $\Delta F / F_{0}$ and $X$ values were pooled; the sensitivity index $d^{\prime}$ and the response bias $\beta$ were estimated from these pooled matrices. The Weber fraction JND\% was then computed from $d^{\prime}$ values across $\Delta F / F_{0}$ values tested for the same $F_{0}$ and $X$ values.

As was the case for compliance discrimination in Experiment $2 \mathrm{~A}$, roving displacement had a strong degrading effect on force discrimination (Figure 8). New data for fixed-displacement paradigm were collected for these same subjects for comparison. The resulting force $\mathrm{JND} \%$, with the mean value shown as a dotted line, was roughly

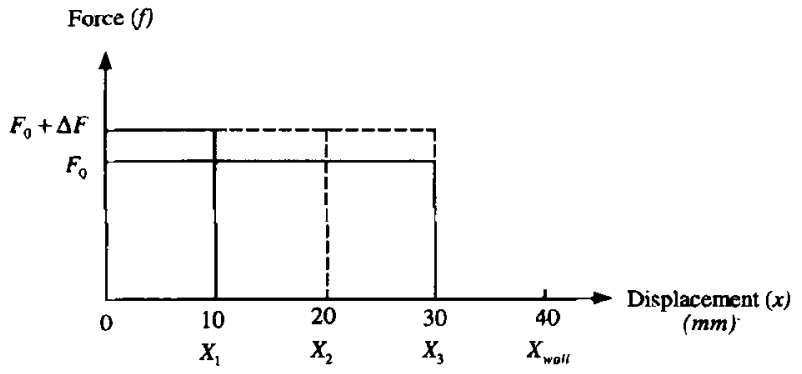

Figure 7. Force-displacement profile for Experíment 2B. Solid lines show two stimuli with $F_{0}$ and $F_{0}+\Delta F$ that differ in displacement (10 and $30 \mathrm{~mm}$, respectively). Dotted lines show the rest of the 6 possible force-displacement profiles associated with $F_{0}, F_{0}+\Delta F$, and $X_{i}(i=1$, 2,3).

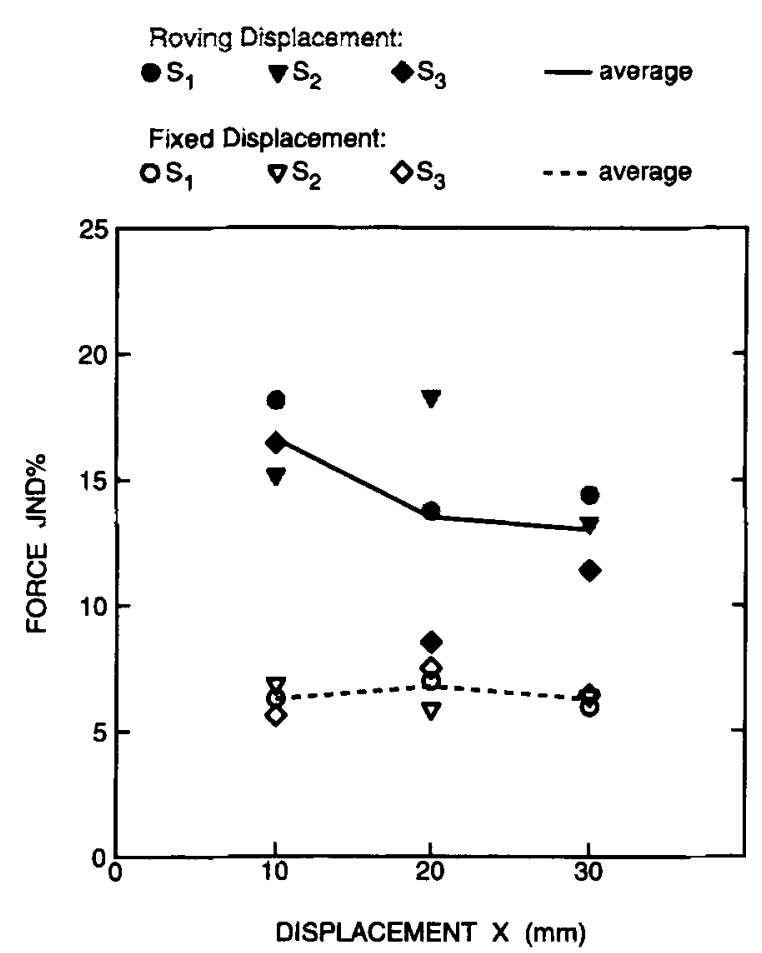

Figure 8. Force IND\% results of Experiment $2 B$.

the same as that found in Pang et al. (1991). Whereas the average force $\mathrm{NND} \%$ for fixed-displacement paradigm was $6 \%$, the average force $\mathrm{JND} \%$ for roving-displacement paradigm increased to $14 \%$. As in Experiments 1 and 2A, the force JND\% values with fixed displacement were essentially independent of $X$, whereas those for roving displacement decreased monotonically with $X$.

The response bias $\beta$ from the fixed-displacement paradigm was consistently small (i.e., $\beta \leq d^{\prime} / 10$ ). Some of the bias data from the roving-displacement paradigm, however, were not negligible and seemed to be a function of displacement $X$ but not $\Delta F / F_{0}$. All bias results for roving displacement from Experiment $2 \mathrm{~B}$ are shown in the right-hand column of Figure 6 . The results of Experiment 2B, like those of Experiment 2A, were reanalyzed according to the work hypothesis. Analysis in terms of the terminal-force hypothesis made no sense here because force was constant during the squeeze.

The work hypothesis and the $d^{\prime}$ results. The work hypothesis for Experiment $2 \mathrm{~B}$ states that the subject responds "smaller force" when $W<W_{T}$ and bigger force when $W \geq$ $W_{T}$, where $W=F \cdot X$, and $W_{T}$ is again chosen to produce the same number of presentations for $W \geq W_{T}$ as for $W<$ $W_{T}$. Table 3 shows the roving-displacement force discrimination data reprocessed according to the work hypothesis in terms of $d^{\prime}$ as a function of $\Delta F / F_{0}$. For comparison, the results obtained by defining correct and incorrect responses according to the force values (normal processing) and pooling the data over the various values of $X$ are also shown. Again, the work hypothesis was strongly supported by S1's data in that (1) the $d^{\prime}$ 'values obtained with 
Table 3

Results of Experiment 2B Reprocessed According to the Work Hypothesis

\begin{tabular}{|c|c|c|c|c|c|c|}
\hline \multirow[b]{2}{*}{$\Delta F / F_{0}$} & \multicolumn{3}{|c|}{$\begin{array}{c}d^{\prime} \\
\text { Work Hypothesis }\end{array}$} & \multicolumn{3}{|c|}{$\begin{array}{c}d^{\prime} \\
\text { Normal Processing } \\
\text { (data pooled over } X \text { ) }\end{array}$} \\
\hline & $\$ 1$ & S2 & S3 & S1 & S2 & S3 \\
\hline $5 \%$ & 1.13 & 0.70 & 0.38 & 0.13 & 0.33 & 0.38 \\
\hline $10 \%$ & 1.49 & 0.54 & 0.76 & 0.64 & 0.53 & 0.82 \\
\hline $15 \%$ & 1.46 & 0.63 & 0.28 & 0.72 & 0.79 & 1.39 \\
\hline $20 \%$ & 1.19 & 0.76 & 0.68 & 1.16 & 1.52 & 1.88 \\
\hline
\end{tabular}

the work hypothesis were higher than those without it, and (2) these $d^{\prime}$ values were approximately constant (the range of mechanical work $W$ was, again, almost constant for the different $\Delta F / F_{0}$ values $^{3}$ ). Results for S2 and S3 were again much less consistent with the work hypothesis. Overall, work seems to have played a somewhat stronger role in the compliance discrimination experiment than in the force discrimination experiment. This was also reflected in the bias data, discussed further below.

The work hypothesis and the $\beta$ results. For the force discrimination experiments, a positive $\beta$ means that the subject tends to respond "larger force" too often. According to the right-hand column in Figure 6, when the displacement $X$ was large, the subjects tended to judge the force to be larger. The biases were also independent of the force increments $\Delta F / F_{0}$. Both observations are consistent with the work hypothesis. Among the 3 subjects, the slope of the $\beta$ versus $X$ curve for $\mathrm{S} 1$ was again the largest.

Summary. The above analyses suggest that, at least for S1, mechanical work might be the underlying common basis for both force and compliance discrimination. The fact that the performance of S1 was much more consistent with the work hypothesis (or the terminal-force hypothesis for Experiment $2 \mathrm{~A}$ ) than that of $\mathrm{S} 2$ or $\mathrm{S} 3$ is consistent with the fact that $\mathrm{S} 1$ did not pay as much attention to the trial-by-trial correct-answer feedback as did $\mathrm{S} 2$ and S3 (information that we obtained from informal observation and conversation with the subjects). To determine whether the elimination of correct-answer feedback based on compliance values would cause other subjects to behave as $\mathrm{S} 1$ behaved in the previously discussed experiments, Experiment $2 \mathrm{~A}$ was repeated over again with new subjects and with the feedback eliminated.

\section{Experiment 2C: Compliance Discrimination Using the Unloaded-Spring, Roving-Displacement Paradigm Without Feedback}

\section{Method}

A new set of subjects (S6, S7, and S8) was tested in this experiment. The subjects served in Experiment $2 \mathrm{~A}$ were not tested again because their response strategy might have been modified by exposure to feedback information. Since no feedback was provided in this experiment, the subjects were given the opportunity to familiarize themselves with the stimuli using the following training procedure. For the first run of 64 trials, the reference compliance $C_{0}$ was kept at $4 \mathrm{~mm} / \mathrm{N}$, the increment $\Delta C / C_{0}$ was set to $0 \%$ (i.e., only $C_{0}$ was presented), and the displacement value for each trial was chosen randomly with an a priori probability of .2 from the following five values: $X_{1}=15, X_{2}=20, X_{3}=25, X_{4}=30$, and $X_{5}=$ $35 \mathrm{~mm}$. The subject was instructed to imagine compressing a linear spring between the thumb and the index finger but was not required to make any judgment. For the second run, $C_{0}$ was increased to $8 \mathrm{~mm} / \mathrm{N}$ and all other parameters were kept the same. The subjects were told that the springs in the first run were stiffer than those in the second run. Starting with the third run, $C_{0}$ was again kept at $4 \mathrm{~mm} / \mathrm{N}, \Delta C / C_{0}$ was set at $100 \%$ (i.e., the compliance values of the first two runs were used here), and the displacement was randomized in the same way as in the previous two runs. The subject was instructed to imagine compressing a linear spring and to judge the compliance of the spring. Each subject was required to complete two runs with $100 \%$ correct scores before the training was terminated. The subjects were able to reach the $100 \%$ correct performance criterion within two or three runs with the third set of stimuli. At no time during training was trial-by-trial feedback given.

For data collection, the procedure was the same as that described in the Method section of Experiment 2A except, of course, that no feedback was provided in this experiment.

\section{Results}

The data were processed in the same way as in Experiment $2 \mathrm{~A}$. The results, shown in Figure 9, indicate once again that roving displacement had a strong degrading effect on the ability to discriminate compliance (S1's data from Experiment $2 \mathrm{~A}$ were plotted again for comparison but were not used for computing the average JND\%). The average compliance $\mathrm{JND} \%$ was, again, $22 \%$. As in Experiment $2 \mathrm{~A}$, the compliance JND\% decreased monotonically with $X$.

When these results were reanalyzed using the work hypothesis and the terminal-force hypothesis (see Table 4 for $d^{\prime}$ results and Figure 10 for $\beta$ results; data for S1 from Experiment 2A are included for comparison), most of

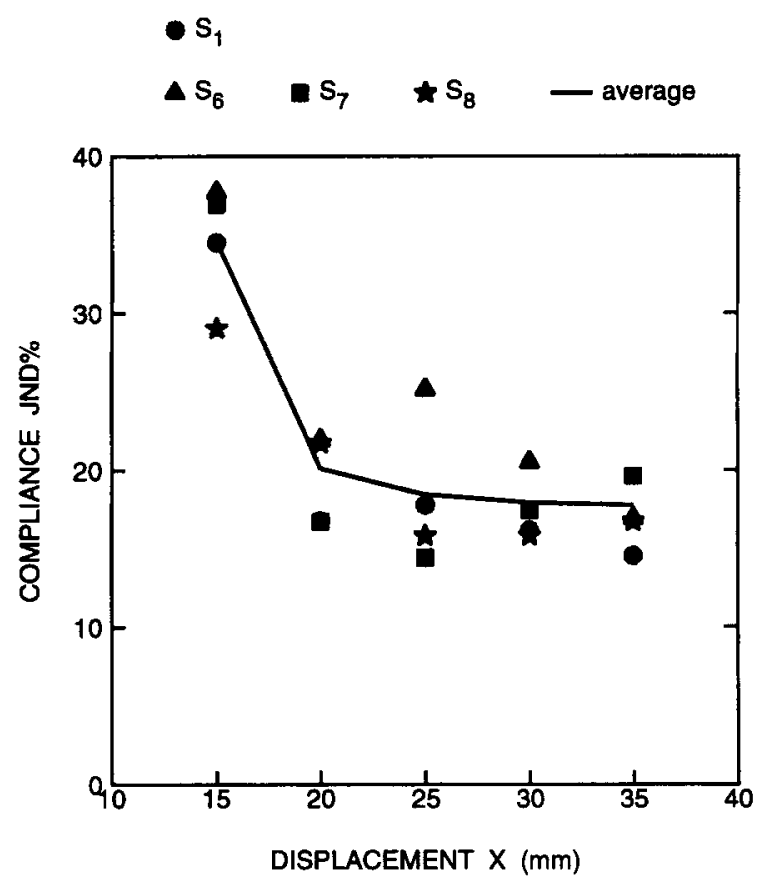

Figure 9. Compliance JND\% results of Experiment 2C. S1's data from Experiment $2 \mathrm{~A}$ are plotted again for comparison. 
Table 4

Results of Experiment 2C Reprocessed According to the Work Hypothesis or the Terminal-Force Hypothesis

\begin{tabular}{|c|c|c|c|c|c|c|c|c|}
\hline \multirow[b]{2}{*}{$\Delta C / C_{0}$} & \multicolumn{4}{|c|}{$\begin{array}{c}d^{\prime} \\
\text { Either Hypothesis }\end{array}$} & \multicolumn{4}{|c|}{$\begin{array}{c}d \\
\text { Normal Processing } \\
\text { (data pooled over } X \text { ) }\end{array}$} \\
\hline & S6 & $\mathrm{S} 7$ & $\mathrm{~S} 8$ & $S I$ & S6 & S7 & S8 & $S 1$ \\
\hline $10 \%$ & 1.54 & 1.60 & 1.12 & 1.63 & 0.52 & 0.33 & 0.51 & 0.26 \\
\hline $20 \%$ & 1.69 & 1.63 & 1.30 & 1.47 & 0.63 & 1.11 & 0.81 & 0.86 \\
\hline $30 \%$ & 1.05 & 1.33 & 1.16 & 1.60 & 1.01 & 1.25 & 1.36 & 1.05 \\
\hline $40 \%$ & 1.15 & 1.48 & 0.91 & 1.41 & 1.14 & 1.46 & 1.48 & 1.65 \\
\hline
\end{tabular}

the data supported these hypotheses in that (1) the $d^{\prime}$ values obtained with these hypotheses were higher than those without them (except for S8's $d^{\prime}$ 's at $\Delta C / C_{0}=30 \%$ and $40 \%),(2)$ these $d^{\prime}$ values were approximately constant, and (3) the $\beta$ versus $X$ curves indicate that when the displacement was small, the subjects tended to judge the object being squeezed as more compliant-a finding that is consistent with both hypotheses. The tendency for S6-S8 to show a smaller value of $|\beta|$ at the largest displacement than at the smallest remains unexplained.

Generally speaking, the results of this experiment confirm our speculation that the difference among the subjects in Experiment $2 \mathrm{~A}$ was caused mainly by the extent to which they attended to the trial-by-trial feedback information. The results from Experiments $2 \mathrm{~A}$ and $2 \mathrm{C}$ suggest that when subjects are not influenced by trial-bytrial correct-answer feedback, they tend to use mechanical work and/or terminal force as the basis for compliance discrimination. The results from Experiment $2 \mathrm{~B}$ suggest that at least one subject (S1) tended to use mechanical work for force discrimination. If mechanical work was the common basis upon which people discriminate compliance and force, then eliminating the work cues in a compliance discrimination task should dramatically degrade subjects' performance. This was the motivation for the design of Experiment 3.

EXPERIMENT 3
Compliance Discrimination Using the Fixed-
Displacement, Equal-Work Paradigm With Feedback
Experiment 3 attempted to measure compliance JNDs
without providing any mechanical-work cues. In order to
equalize work for two different compliance values, it was
necessary to vary the initial-force offset and terminal-force
cues with compliance cues (this was true under the con-
straints of a linear spring simulation and a fixed-
displacement paradigm). By carefully choosing the pa-
rameters for compliance discrimination, however, it was
possible to keep the initial- and terminal-force increments
much smaller than the compliance increment. Thus, the

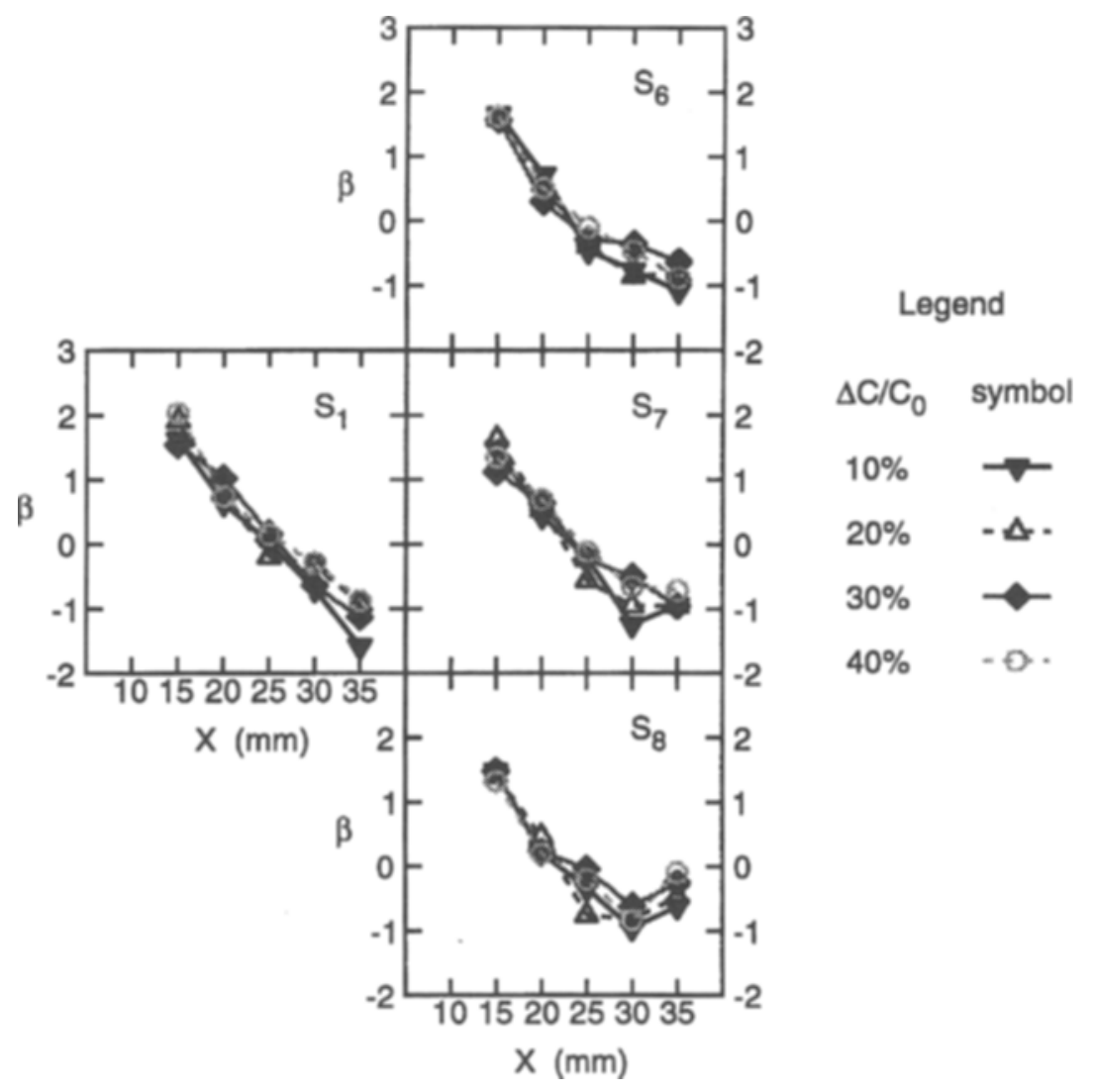


subjects in this experiment had little information to work with other than the compliance information.

\section{Method}

A different set of subjects (S2, S4, and S5) participated in this experiment. Only $\mathrm{S} 2$ participated in some of the previous experiments. The subject was instructed to imagine compressing a linear spring between the thumb and the index finger and to judge the compliance of the spring. The fixed-displacement paradigm was employed and trial-by-trial correct-answer feedback based on compliance values was provided. The force-displacement profile simulated preloaded linear springs (Figure 11). The resistive force $f$ was related to displacement $x$ by $f=F_{M}+(x-X / 2) / C$, where $F_{M}$ denoted the midpoint force and $C$ was either $C_{0}$ or $C_{0}+\Delta C$. In this equal-work paradigm, the mechanical work cue was totally eliminated (i.e., the two stimuli being discriminated required the same amount of work to squeeze). The initial- and terminal-force cues could be reduced by increasing the midpoint force $F_{M}$ without changing the reference compliance $C_{0}$, the compliance increment $\Delta C / C_{0}$, or the displacement $X$. For example, for $F_{M}=6 \mathrm{~N}, X=20 \mathrm{~mm}, C_{0}=$ $4 \mathrm{~mm} / \mathrm{N}$, and $\Delta C / C_{0}=20 \%$, the terminal-force increment $\Delta F / F_{0}$ was $4.9 \%$. By increasing $F_{M}$ to $9 \mathrm{~N}$ without changing any other parameters, $\Delta F / F_{0}$ was reduced to $3.6 \%$. Note that these terminal-force increments were much smaller than the compliance increment and were below the average JND $\%(6 \%-7 \%)$ obtained from force discrimination experiments with fixed displacement (Pang et al., 1991; see also the dotted line in Figure 8). Therefore, if the subject could discriminate the two stimuli well (i.e., if $d^{\prime} \gg 1$ ), then they must have relied mainly on compliance cues. Given the above parameter settings, however, if the subject relied on other extraneous cues for compliance discrimination, then the performance would be poor (i.e., $d^{\prime}<1$ ).

Table 5 shows the experimental conditions tested. Instead of using fixed $\Delta \mathrm{C} / \mathrm{C}_{0}$ values for all conditions, four compliance increments were chosen for each condition such that the percent correct scores varied from $\approx 60 \%$ to $\approx 90 \%$. The same $\Delta C / C_{0}$ values were used for all 3 subjects. Only $X=10 \mathrm{~mm}$ was used with $C_{0}=$ $2 \mathrm{~mm} / \mathrm{N}$, because the terminal force associated with 20 or $30 \mathrm{~mm}$ was too great for the subjects to keep squeezing without becoming fatigued (e.g., $F_{0}=14 \mathrm{~N}$ for $C_{0}=2 \mathrm{~mm} / \mathrm{N}, X=20 \mathrm{~mm}$, and $F_{M}=$ $9 \mathrm{~N})$. Note also that for $C_{0}=4 \mathrm{~mm} / \mathrm{N}$ and $X=30 \mathrm{~mm}, F_{M}$ started from $6 \mathrm{~N}$ so that the initial-force offset was positive (e.g., the initial-force offset would be $-0.75 \mathrm{~N}$ for $F_{M}=3 \mathrm{~N}$ ). There were a total of 29 experimental conditions. Each subject performed 8 runs per condition ( 2 runs per $\Delta C / C_{0}$ value). The order of the experimental conditions was randomized for each subject.

\section{Results}

As in the previous experiments, the stimulus-response matrices from the same experimental condition were

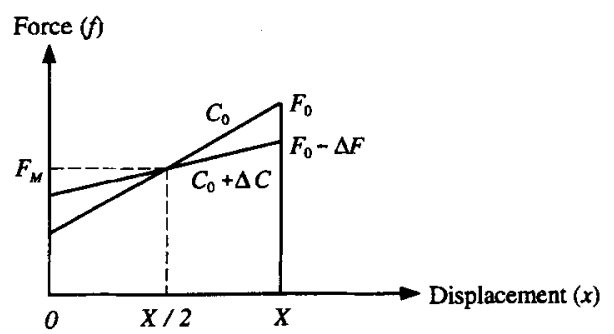

Figure 11. Force-displacement profile for Experiment 3. $C_{0}$ is the reference compliance, $\Delta C$ is the compliance increment, $F_{0}$ is the terminal force associated with $C_{0}, \Delta F$ is the terminal-force increment, $F_{M}$ is the midpoint force, and $X$ is the total displacement. pooled. The sensitivity index $d^{\prime}$ and the response bias $\beta$ were estimated. The Weber fraction JND\% was then computed from $d^{\prime}$ values across $\Delta C / C_{0}$ values tested for the same $C_{0}, X$, and $F_{M}$ values. The bias $\beta$ values were negligible compared to $d^{\prime}$ (i.e., $\beta \leq d^{\prime} / 10$ ). Therefore, the JND\% results completely characterized the data.

Values of the measured compliance JND\% are plotted in Figure 12. Most of these values are much larger than the force and compliance JND\%s obtained earlier. Individual subject's JND\%s varied from $11.6 \%\left(\mathrm{~S} 4: C_{0}=\right.$ $4 \mathrm{~mm} / \mathrm{N}, X=30 \mathrm{~mm}$, and $\left.F_{M}=6 \mathrm{~N}\right)$ to $136.5 \%\left(\mathrm{~S} 2: C_{0}=\right.$ $8 \mathrm{~mm} / \mathrm{N}, X=10 \mathrm{~mm}$, and $\left.F_{M}=9 \mathrm{~N}\right)$. The average JND $\%$ varied from $15 \%\left(C_{0}=4 \mathrm{~mm} / \mathrm{N}, X=30 \mathrm{~mm}\right.$, and $\left.F_{M}=6 \mathrm{~N}\right)$ to $99 \%\left(C_{0}=8 \mathrm{~mm} / \mathrm{N}, X=10 \mathrm{~mm}\right.$, and $F_{M}=$ $9 \mathrm{~N})$.

It was not immediately apparent how the compliance JND\%s plotted in Figure 12 could be summarized; however, three trends were observed. First, given $C_{0}$ and $X$, the compliance JND\% increased monotonically with $F_{M}$ (see Figure 12). Second, given $C_{0}$ and $F_{M}$, the compliance JND\% decreased monotonically with $X$ (see Figure 13, in which the results from different subjects are averaged). Third, given $F_{M}$ and $X$, the compliance JND\% increased monotonically with $C_{0}$ (see Figure 14, in which the results from different subjects are averaged). On the basis of these trends, it was speculated that the wide range of compliance $\mathrm{NND} \% \mathrm{~s}$ might be related to the changes in terminal-force increments over the wide range of test conditions. In order to explore this hypothesis, the following computation was made.

For each subject and each of the 29 experimental conditions, the compliance JND $\% \mathrm{~s},(\Delta C)_{0} / C_{0}$, were converted to the corresponding terminal-force JND $\%$ s, $(\Delta F)_{0} / F_{0}$, in the following way. From Figure 11,

$$
F_{0}=F_{M}+\frac{X}{2 C_{0}}
$$

and

$$
F_{0}-(\Delta F)_{0}=F_{M}+\frac{X}{2\left[C_{0}+(\Delta C)_{0}\right]}
$$

Thus,

$$
(\Delta F)_{0}=\frac{X}{2}\left(\frac{1}{C_{0}}-\frac{1}{C_{0}+(\Delta C)_{0}}\right)=\frac{X}{2 C_{0}}\left(\frac{(\Delta C)_{0} / C_{0}}{1+(\Delta C)_{0} / C_{0}}\right) .
$$

Both $(\Delta F)_{0}$ and $F_{0}$ can be expressed in terms of the known parameters $F_{M}, X$, and $C_{0}$, and the compliance $\mathrm{JND} \%$ $(\Delta C)_{0} / C_{0}$. Therefore, terminal-force $\mathrm{JND} \%$ can be calculated as $(\Delta F)_{0} / F_{0}$. A total of $87(3$ subjects $\times 29$ conditions) $(\Delta F)_{0} / F_{0}$ values were computed and averaged. The result was surprisingly simple:

$$
(\Delta F)_{0} / F_{0}=5.15 \% \pm 0.76 \% \text {. }
$$

The average terminal-force $\mathrm{JND} \%$ of $5.15 \%$ was roughly consistent with the average JND\% we obtained earlier from Experiment 2B with fixed displacement. Also, the standard deviation of $0.76 \%$ was small, especially considering the fact that the average $(\Delta C)_{0} / C_{0}$ varied from 
Table 5

Experimental Conditions for Experiment 3

\begin{tabular}{|c|c|c|c|}
\hline $\begin{array}{c}\text { Reference } \\
\text { Compliance } \\
C_{0}(\mathrm{~mm} / \mathrm{N}) \\
\end{array}$ & $\begin{array}{c}\text { Displacement } \\
X(\mathrm{~mm}) \\
\end{array}$ & $\begin{array}{c}\text { Midpoint Force } \\
F_{M}(\mathrm{~N})\end{array}$ & $\begin{array}{c}\text { Compliance } \\
\text { Increments } \\
\Delta C / C_{0}(\%)\end{array}$ \\
\hline \multirow[t]{3}{*}{2} & 10 & 4 & $10,20,30,40$ \\
\hline & & 6 & $20,30,40,50$ \\
\hline & & 9 & $20,40,60,80$ \\
\hline \multirow[t]{8}{*}{4} & 10 & 3 & $20,30,40,50$ \\
\hline & & 6 & $20,40,60,80$ \\
\hline & & 9 & $25,50,75,100$ \\
\hline & 20 & 4 & $10,20,30,40$ \\
\hline & & 6 & $20,30,40,50$ \\
\hline & & 9 & $20,40,60,80$ \\
\hline & 30 & 6 & $10,20,30,40$ \\
\hline & & 9 & $20,30,40,50$ \\
\hline \multirow[t]{9}{*}{6} & 10 & 3 & $20,40,60,80$ \\
\hline & & 6 & $25,50,75,100$ \\
\hline & & 9 & $30,60,90,120$ \\
\hline & 20 & 4 & $20,30,40,50$ \\
\hline & & 6 & $20,40,60,80$ \\
\hline & & 9 & $25,50,75,100$ \\
\hline & 30 & 4 & $10,20,30,40$ \\
\hline & & 6 & $20,30,40,50$ \\
\hline & & 9 & $20,40,60,80$ \\
\hline \multirow[t]{9}{*}{8} & 10 & 3 & $25,50,75,100$ \\
\hline & & 6 & $30,60,90,120$ \\
\hline & & 9 & $40,80,120,160$ \\
\hline & 20 & 3 & $20,30,40,50$ \\
\hline & & 6 & $20,40,60,80$ \\
\hline & & 9 & $50,75,100,125$ \\
\hline & 30 & 4 & $10,20,30,40$ \\
\hline & & 7 & $20,30,40,50$ \\
\hline & & 10 & $20,40,60,80$ \\
\hline
\end{tabular}

$15 \%$ to $99 \%$. Thus, it seemed that when no work cues were available, the subjects tended to rely on terminal-force cues to perform compliance discrimination tasks.

From Figure 11, it is obvious that the initial-force increment was always equal to the corresponding terminalforce increment $\Delta F$ in these experiments. Therefore, the initial-force $\mathrm{JND} \%$ was related to the terminal-force $\mathrm{NND} \%$ by the ratios of terminal over initial forces associated with the reference compliance. This ratio ranged from 1.15 to 4.33 across experimental conditions. Therefore, it was not possible to obtain a single number with a small standard deviation for the initial-force JND\%s. It thus seemed unlikely that the initial-force cues played a major role in compliance discrimination under these conditions.

\section{DISCUSSION}

Our experiments were designed to measure the compliance JND and to investigate the possible roles of force and mechanical-work cues in compliance discrimination. Overall, our results suggest that people tend to use mechanical work and/or terminal-force cues for compliance discrimination whenever such cues are available (see Table 6). A parsimonious description of results from all compliance experiments (i.e., all experiments except Experiment $2 \mathrm{~B}$ ) is that the subjects based their judgments on terminal-force values rather than on compliance val- ues. Moreover, all the results of the compliance experiments can be predicted by assuming a terminal-force JND of 5\%-7\%. Alternatively, the results from all experiments except Experiment 3 are consistent with the hypothesis that subjects based their judgments on the amount of mechanical work rather than compliance or force values. However, because mechanical-work JNDs are not available for comparison, it is not clear whether the results from all experiments except Experiment 3 can be readily predicted from mechanical-work JNDs. At this point, our results do not permit us to strongly favor the terminalforce hypothesis over the mechanical-work hypothesis, or vice versa. We can only conclude that people tend to use terminal-force and/or mechanical-work cues for compliance discrimination whenever such cues are available. In general, compliance JNDs appear to be considerably larger than force JNDs, and the precise values of these JNDs depend upon the particular experimental paradigm used for testing (see Table 6).

Perception of force and compliance (or, equivalently, stiffness, the inverse of compliance) has been investigated by several researchers (Harper \& Stevens, 1964; Jones, 1989; Jones \& Hunter, 1990; Pang et al., 1991; Roland \& Ladegaard-Pedersen, 1977; Srinivasan \& LaMotte, 1994). Table 7 summarizes the results of these studies. Harper and Stevens (1964) studied magnitude estimation of apparent hardness and softness using specimens with 


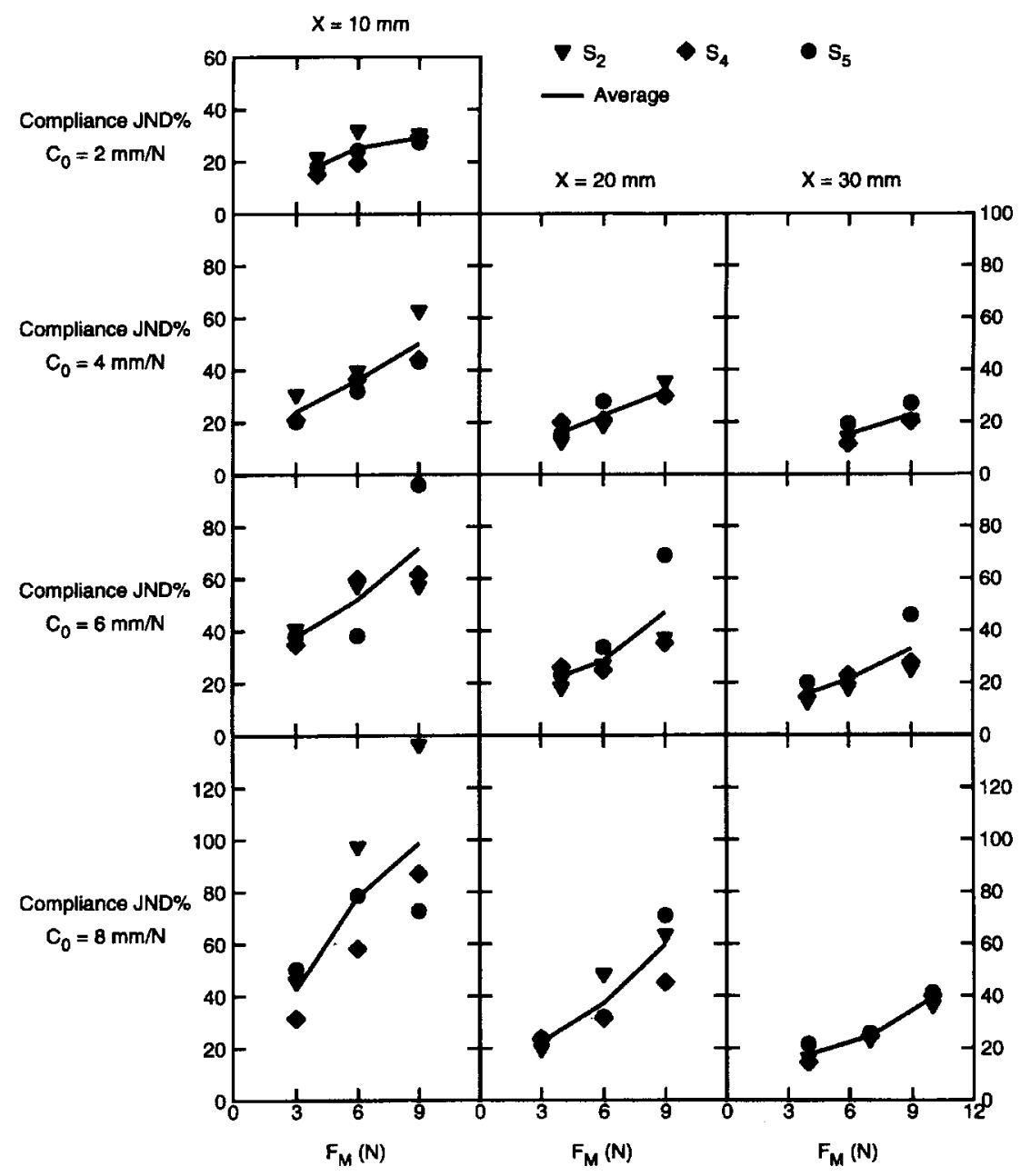

Figure 12. Compliance JND\% results of Experiment 3.

different physical hardness (force/indentation). Their data supported the psychophysical power law and showed that subjective hardness and softness grew as the physical hardness raised to a power of exponent 0.8 and -0.8 , respectively. These results indicate that (1) people are capable of making magnitude judgments of both hardness and softness and (2) the two sensations are equally spaced in the perceptual domain (i.e., if object $A$ is judged to be twice as hard as object $B$, then $B$ is judged to be twice as soft as A). Thus, studies of stiffness and compliance should yield similar results. Roland and Ladegaard-Pedersen (1977) measured discrimination limits (i.e., JND\%) on the stiffness of springs (contained in cylinders with rigid surfaces) compressed between the thumb and the index finger. In their experiments, the subject always started with an unloaded spring, and the lengths of the springs were chosen such that a force of $9.80 \mathrm{~N}$ gave the maximum displacement. In other words, they employed an unloadedspring, equal terminal-force paradigm with roving displacement. Their stiffness JND\% of $17 \%$ with unanesthetized fingers was higher than that from Experiment 1 $(8 \%)$ but lower than that from Experiment $2 \mathrm{~A}(22 \%)$. Con- sider now the results on stiffness perception obtained by Jones and Hunter (1990). In their experiments, the stiffness JND was estimated using a contralateral limbmatching procedure in which subjects adjusted the stiffness of the motor connected to one wrist until it was perceived to be the same as that connected to the other wrist by moving the forearm about the elbow joint in the vertical plane. Their results, like ours, showed relatively poor performance: the estimated JND was roughly $23 \%$. Although it is impossible to make precise comparisons between their results and ours because of the substantial differences in experimental methodology and the muscle group activated, it seems most reasonable to compare their results (obtained from experiments in which the displacement was essentially uncontrolled) with the results we obtained with roving displacement (i.e., to our JND of roughly $22 \%$ ). In her force discrimination experiments (Jones, 1989), the force JND was estimated to be $7 \%$ by a contralateral limb-matching procedure in which subjects isometrically contracted the elbow flexor muscles of both arms. Because there was effectively no displacement in these experiments, it is not surprising 


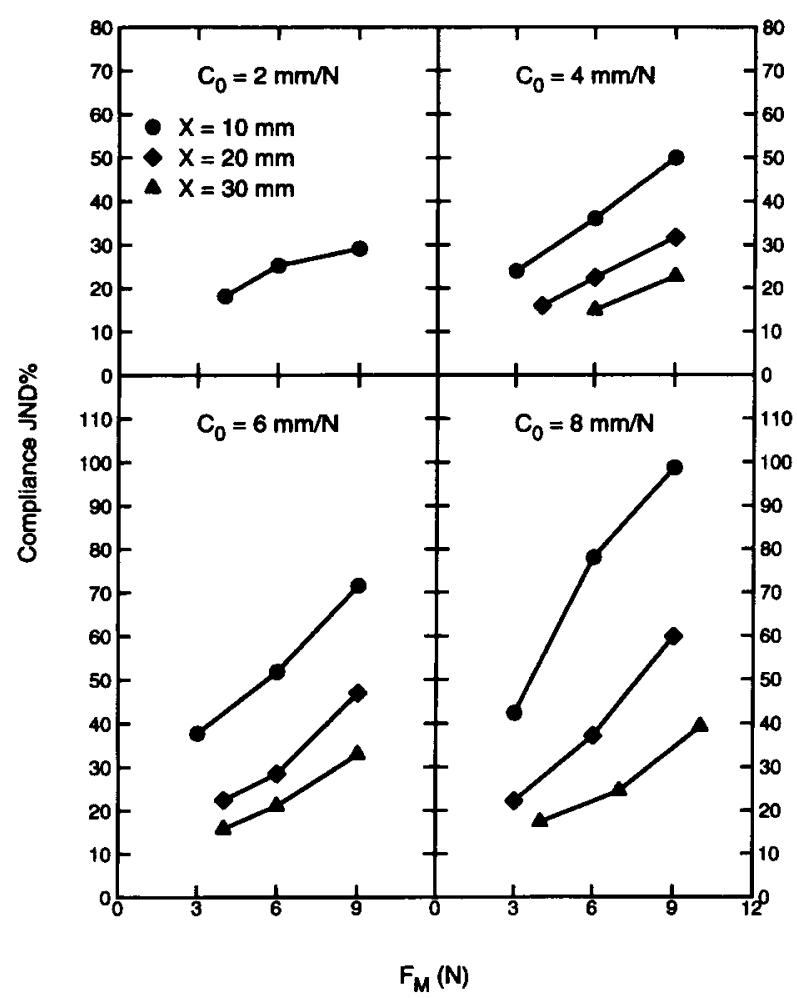

Figure 13. Average compliance JND\% results of Experiment 3. The average data from Figure 12 are replotted here to show the effect of displacement $X$ on compliance $J \mathrm{ND} \%$.

that the force JNDs obtained by Jones (1989) are comparable to the force JNDs that we obtained in Experiment $2 \mathrm{~B}$ using the fixed-displacement paradigm (i.e., $6 \%$, see also Pang et al., 1991).

Most studies to date on kinesthesis have focused on position and force (Clark \& Horch, 1986). How people estimate compliance, stiffness, or mechanical work from position and force cues is still not clear. It seems reasonable to assume, however, that people do possess the ability to attend to these variables based on position and force information. Other researchers (e.g., Solomon \& Turvey, 1988) have shown that our ability to estimate reachable distance through a hand-held rod was dependent on the principal moment of inertia of the hand-rod system, even when the moments of inertia in question were components of the total moments of inertia of the rods. Thus, people presumably have some way of estimating moments of inertia as well. Characterizing discrimination resolution of these perceptual variables is a first step toward the understanding of the neural computations involved.

Finally, in considering our results and their relationship to previous data or to theoretical models, the following points should be noted. First, our results clearly show that randomizing the displacement from trial to trial has a substantial degrading effect on both force and compliance discrimination. That this result indicates a specific interaction between displacement and force or between displacement and compliance (rather than a general high-level distraction effect) is supported not only by the systematic characteristics of the effects observed (incorporated in the terminal-force and work hypotheses) but also by the existence of many pairs of psychophysical variables that are known to be perceptually separable (e.g., see Ashby \& Townsend, 1986; Garner, 1974). Second, our results suggest that the elevated JND values found for compliance cannot be explained simply in terms of the need for the subject to perform the operation of division to estimate compliance (i.e., the need to divide change in force by change in displacement to estimate the slope of the force vs. displacement profile) when terminal-force and work cues are eliminated. Although it is possible that such a model would fit the data on compliance (as well, perhaps, as viscosity), it would leave totally unexplained the degrading effects of roving displacement on force discrimination. Third, in our experiments, as well as those of Roland and LadegaardPedersen (1977), the use of rigid surfaces for interfacing the fingers to the compliant object influences the relative importance of the roles played by the tactile system and the kinesthetic/proprioceptive system in discriminating compliance. In particular, the tactile information derived from local deformation of the fingerpads is less important in these experiments than in those in which the object surface also reflects the compliance value (see Srinivasan \& LaMotte, 1994, and our comments in the introduction

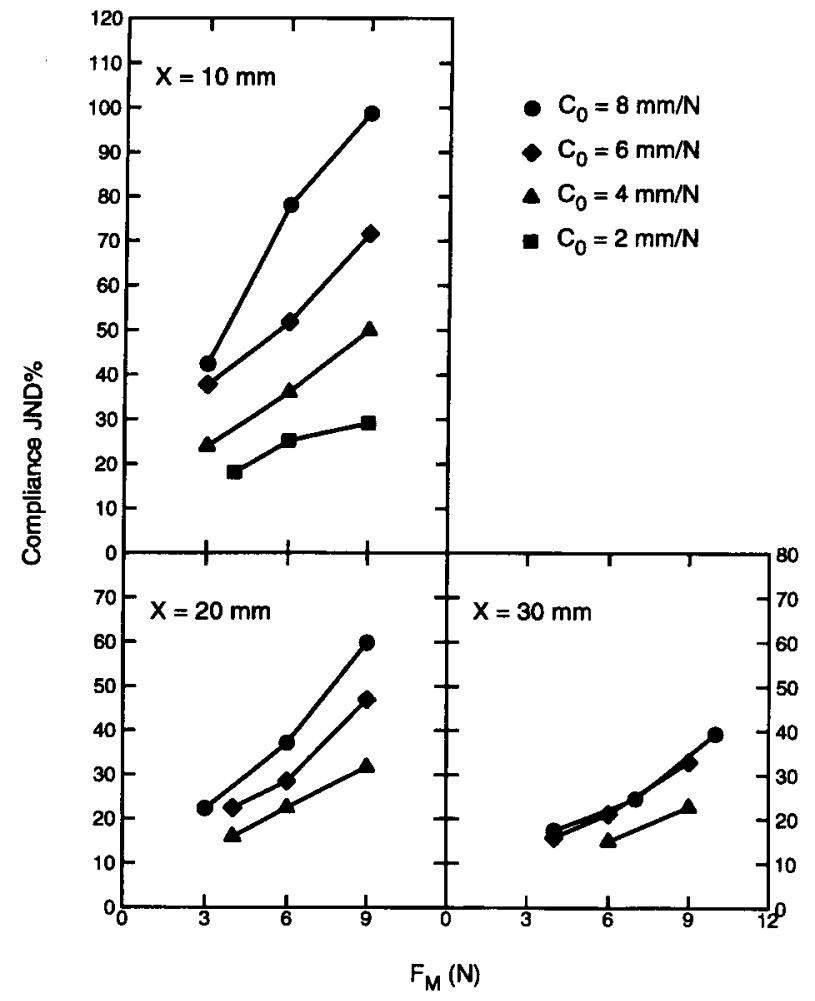

Figure 14. Average compliance JND\% results of Experiment 3. The average data from Figure 12 are replotted here to show the effect of reference compliance $C_{0}$ on compliance JND $\%$. 
Table 6

Summary of Results of This Study

\begin{tabular}{|c|c|c|c|c|c|}
\hline Experiment & $\begin{array}{c}\text { Target } \\
\text { Parameter }\end{array}$ & Paradigm & Possible Cues & $\begin{array}{l}\text { Average } \\
\text { JND } \% \text { of } \\
\text { Target }\end{array}$ & $\beta$ \\
\hline 1 & compliance & $\begin{array}{l}\text { unloaded spring, } \\
\text { fixed displacement } \\
\text { with feedback }\end{array}$ & $\begin{array}{l}\text { mechanical work } \\
\& \text { terminal force }\end{array}$ & $8 \%$ & negligible \\
\hline $2 \mathrm{~A}$ & compliance & $\begin{array}{l}\text { unloaded spring, } \\
\text { roving displacement } \\
\text { with feedback }\end{array}$ & $\begin{array}{l}\text { mechanical work } \\
\& \text { terminal force } \\
\text { (S1's data) }\end{array}$ & $22 \%$ & $\begin{array}{l}\text { dependent on } \\
\text { displacement }\end{array}$ \\
\hline $2 B$ & force & $\begin{array}{l}\text { roving displacement } \\
\text { with feedback }\end{array}$ & $\begin{array}{l}\text { mechanical work } \\
\text { (S1's data) }\end{array}$ & $14 \%$ & $\begin{array}{l}\text { dependent on } \\
\text { displacement }\end{array}$ \\
\hline $2 \mathrm{C}$ & compliance & $\begin{array}{l}\text { unloaded spring, } \\
\text { roving displacement } \\
\text { without feedback }\end{array}$ & $\begin{array}{l}\text { mechanical work } \\
\& \text { terminal force }\end{array}$ & $22 \%$ & $\begin{array}{l}\text { dependent on } \\
\text { displacement }\end{array}$ \\
\hline 3 & compliance & $\begin{array}{c}\text { fixed displacement, } \\
\text { equal work } \\
\text { with feedback }\end{array}$ & $\begin{array}{l}\text { terminal force } \\
\quad(\mathrm{JND} \%= \\
5.15 \pm 0.76 \%)\end{array}$ & $15 \%-99 \%$ & negligible \\
\hline
\end{tabular}

Table 7

Summary of Studies on the Perception of Force and Stiffness

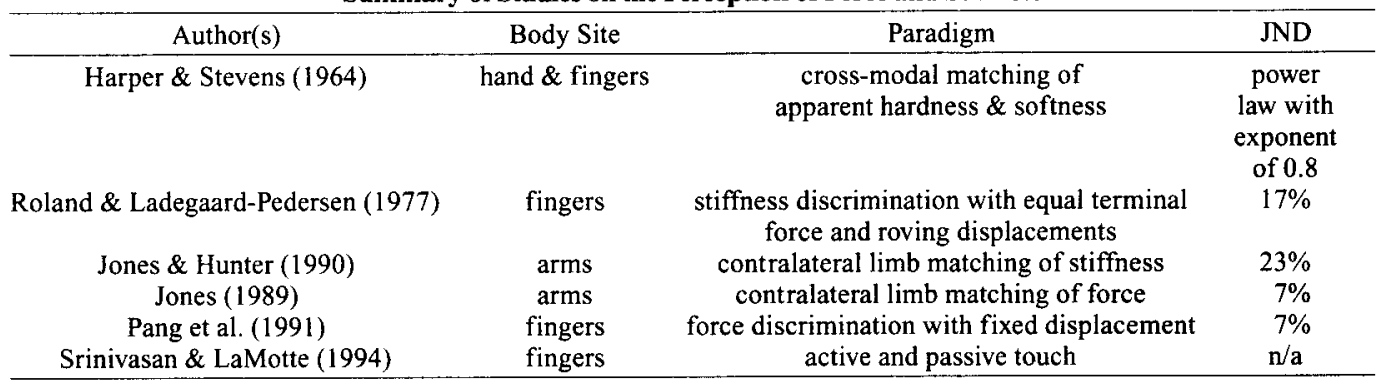

to this paper). Inasmuch as the perception of work relates to muscle action rather than fingerpad deformation, one would expect the work hypothesis to be less relevant to the latter group of experiments than to the former.

Future work in our research program will include measurements of the mechanical work JND as well as measurements of the displacement JND with roving force and compliance. In addition, experiments will be conducted on the ability to discriminate viscosity and mass and on the ability to resolve combinations of force, compliance, viscosity, and mass into constituent components.

\section{REFERENCES}

Ashby, F. G., \& Townsend, J. T. (1986). Varieties of perceptual independence. Psychological Review, 93, 154-179.

BerLiner, J. E., \& DURLACH, N. I. (1973). Intensity perception IV: Resolution in roving-level discrimination. Journal of the Acoustical Society of America, 53, 1270-1287.

Clark, F. J., Burgess, R. C., \& Chapin, J. W. (1986). Proprioception with the proximal interphalangeal joint of the index finger: Evidence for a movement sense without a static-position sense. Brain, 109, $1195-1208$.

Clark, F. J., \& Horch, K. W. (1986). Kinesthesia. In K. R. Boff, L. Kaufman, \& J. P. Thomas (Eds.), Handbook of perception and human performance: Sensory processes and perception (pp. 13/113/62). New York: Wiley.

Durlach, N. I., Delhorne, L. A., Wong, A., Ko, W. Y., Rabinowitz, W. M., \& HollerbaCH, J. (1989). Manual discrimination and iden- tification of length by the finger-span method. Perception \& Psychophysics, 46, 29-38.

DurLach, N. I., \& Mavor, A. S. (Eds.). (1994). Virtual reality: Scientific and technological challenges. Washington, DC: National Academy Press.

FERRELL, W. R., \& MiLnE, S. E. (1989). Factors affecting the accuracy of position matching at the proximal interphalangeal joint in human subjects. Journal of Physiology, 411, 575-583.

GARNER, W. R. (1974). The processing of information and structure. Potomac, MD: Erlbaum.

HARPER, R., \& STEVENS, S. S. (1964). Subjective hardness of compliant materials. Quarterly Journal of Experimental Psychology, 16, 204-215.

JoNES, L. A. (1986). Perception of force and weight: Theory and research. Psychological Bulletin, 100, 29-42.

JoNES, L. A. (1989). Matching forces: Constant errors and differential thresholds. Perception, 18, 681-687.

JONES, L. A., \& HUNTER, I. W. (1990). A perceptual analysis of stiffness. Experimental Brain Research, 79, 150-156.

Jones, L. A., \& Hunter, I. W. (1992a). Differential thresholds for limb movement measured using adaptive techniques. Perception \& Psychophysics, 52, 529-535.

JONES, L. A., \& HUNTER, I. W. (1992b). Human operator perception of mechanical variables and their effects on tracking performance. Winter Annual Meeting of the American Society of Mechanical Engineers: Advances in Robotics, 42, 49-53.

JONES, L. A., \& HUNTER, I. W. (1993). A perceptual analysis of viscosity. Experimental Brain Research, 94, 343-351.

Pang, X. D., TAN, H. Z., \& Durlach, N. I. (1991). Manual discrimination of force using active finger motion. Perception \& Psychophysics, 49, 531-540.

Roland, P. E., \& Ladegaard-Pedersen, H. (1977). A quantitative 
analysis of sensations of tension and of kinaesthesia in man: Evidence for a peripherally originating muscular sense and for a sense of effort. Brain, 100, 671-692.

Solomon, H. Y., \& TuRveY, M. T. (1988). Haptically perceiving the distances reachable with hand-held objects. Journal of Experimental Psychology: Human Perception \& Performance, 14, 404. 427.

SRINIVASAN, M. A., \& LAMotTE, R. H. (1994). Tactual discrimination of softness. Journal of Neurophysiology, 73, 88-101.

TAN, H. Z., Durlach, N. I., Shao, Y., \& WeI, M. (1993). Manual resolution of compliance when work and force cues are minimized. Winter Annual Meeting of the American Society of Mechanical Engineers: Advances in Robotics, Mechatronics, \& Haptic Interfaces, 49, 99-104.

TAN, H. Z., PANG, X. D., \& DuRlach, N. I. (1992). Manual resolution of length, force, and compliance. Winter Annual Meeting of the American Society of Mechanical Engineers: Advances in Robotics, 42, 13-18.

Watson, J. D. G., Colebatch, J. G., \& McCloskey, D. I. (1984). Effects of externally imposed elastic loads on the ability to estimate position and force. Behavioral Brain Research, 13, 267-271.

\section{NOTES}

1. $1 \mathrm{~N}=1$ newton $=1 \mathrm{~kg} \cdot \mathrm{m} / \mathrm{sec}^{2}$. It is equal to the weight of $102 \mathrm{~g}$ (or $0.225 \mathrm{lb}$ ) of mass.

2. Tactual sensory information from the hand in contact with an object can be divided into two classes: (1) tactile information, referring to the sense of contact with the object, mediated by the responses of low-threshold mechanoreceptors innervating the skin (say, the fingerpad) within and around the contact region, and (2) kinesthetic information, referring to the sense of position and motion of limbs along with the associated forces, conveyed by the sensory receptors in the skin around the joints, joint capsules, tendons, and muscles, together with neural signals derived from motor commands.

3. The ranges of $W$ for the different $\Delta F / F_{0}$ values were 50 to 157.5 $\mathrm{N} \cdot \mathrm{mm}$ when $\Delta F / F_{0}=5 \% ; 50$ to $165 \mathrm{~N} \cdot \mathrm{mm}$ when $\Delta F / F_{0}=10 \% ; 50$ to $172.5 \mathrm{~N} \cdot \mathrm{mm}$ when $\Delta F / F_{0}=15 \%$; and 50 to $180 \mathrm{~N} \cdot \mathrm{mm}$ when $\Delta F / F_{0}=$ $20 \%$. These values were computed in the same fashion as those for the compliance data except, of course, that the formula for computing work was different. In the force experiment, work was computed according to the formula $W=F \cdot X$, whereas in the compliance experiment, work was computed according to the formula $W=X^{2} /(2 C)$.

(Manuscript received November 8, 1993;

revision accepted for publication November $24,1994$. 